\title{
Nonlinear Distributed Parameter Observer Design for Fuel Cell Systems
}

\author{
Julio Luna*, Attila Husar, Maria Serra \\ Institut de Robòtica i Informàtica Industrial (CSIC-UPC). C/Llorens i Artigas 4-6. 08028 Barcelona, Spain
}

\begin{abstract}
This paper presents the development of a nonlinear state observer to estimate the different gas species concentration profiles in a Proton Exchange Membrane Fuel Cell energy system. The selection of the estimated states follows functionality and fuel cell performance criteria. The implementation is based on the finite element discretisation of a fuel cell distributed parameter model. Forward and backwards discretisation of the partial derivative equations is performed to take advantage of the boundary conditions of the problem and also to apply lumped systems theory in the synthesis procedure of the observer. A second-order sliding-mode super-twisting corrective input action is implemented to reduce the estimation error to zero in a finite amount of time. The sliding-mode control approach grants a suitable corrective action without incrementing the model-dependency of the observer. Simulation results are presented to show the performance of the proposed observer of the fuel cell internal states and to extract conclusions for future research work.
\end{abstract}

Keywords: PEMFC, distributed parameter model, nonlinear observer, super-twisting, observation

\section{Introduction}

Fuel cell technologies are an interesting alternative for clean energy production. Particularly, PEMFC, with high power density, are very promising for mass market applications such as automotive and stationary combined heat and power (CHP) systems. Great research efforts are being dedicated to improve efficiency, reduce degradation and decrease production cost of this technology. In the automatic control field, new estimation, diagnosis and control systems are being developed.

To operate properly, different physical variables have to be measured from the PEMFC, which make it possible to implement output-feedback control laws. While a certain number of these measurements are feasible with the current existing sensor technology, due to the enclosed construction of the system, there are parts that are inaccessible and therefore, state estimation techniques are needed to obtain the internal states values. The internal conditions are a key aspect for the durability, reliability and safety of PEMFC and the overall energy system. Specifically, the different species gas profiles along the PEMFC channels are critical variables for performance.

Nonlinear state observation and unknown input reconstruction is one of the most important problems in modern control theory. Employing a dynamic model representation of the plant, it is possible to implement techniques used in robust control, such as linear matrix inequalities

*Corresponding author. Tel: +34 934015805 - Fax: +34 93 4015750

Email address: jluna@iri.upc.edu (Julio Luna)
(LMI) [1, 2] or sliding-mode control (SMC) [3] to estimate the values of the states.

A limited number of works have been published regarding the nonlinear observation in PEMFC systems aimed to design appropriate controllers and perform diagnosis. Some main ideas can be taken from these works. The membrane water content estimation [4] is a critical aspect of the water management problem, which is crucial when operating a PEMFC. The inlet oxygen flows [5] and fuel cell hydrogen estimation [6] through the measurement of the output manifold pressures are necessary to maintain a proper reactant amount in the system and to decrease the hydrogen consumption respectively.

Model-based nonlinear state observation has an extensive amount of advantages due to the consideration of the nonlinear dynamics of the system. It makes it possible to implement output-feedback nonlinear control techniques that allow the system to operate far away from its nominal working point, which is a common situation in PEMFC energy systems. The main contribution of this paper relies on the implementation of a nonlinear observer topology [7] based on a distributed parameters model [8] of a PEMFC, for the estimation of the concentrations profiles along the channels of all the gas species. The resulting nonlinear distributed parameter observer (NDPO) should contribute to improve the efficiency and life expectancy of PEMFC systems if used to obtain advanced controllers that consider the internal dynamics of the system.

The paper is organized as follows. In Section 2, the general system description and statement of the observation problem are presented. In Section 3 the mathematical model of the PEMFC is presented. The analysis of 
the observed variables is presented in Section 4. Section 5 includes the design of the nonlinear observer. Simulation results are shown in Section 6. Finally, in Section 7, the conclusions of this research work are presented.

\section{System description}

The paper focuses on a single channel PEMFC. A schematic representation of the system is shown in Fig. 1. The single channel PEMFC model used to simulate the state estimation solutions has a z-axis or channel length $(\mathrm{L})$ of $0.4 \mathrm{~m}$ and a $\mathrm{x}$-axis or channel width $\left(\mathrm{L}_{x}\right)$ of $1 \times 10^{-3} \mathrm{~m}$. This gives a total active area of $0.4 \times 10^{-3} \mathrm{~m}^{2}$.

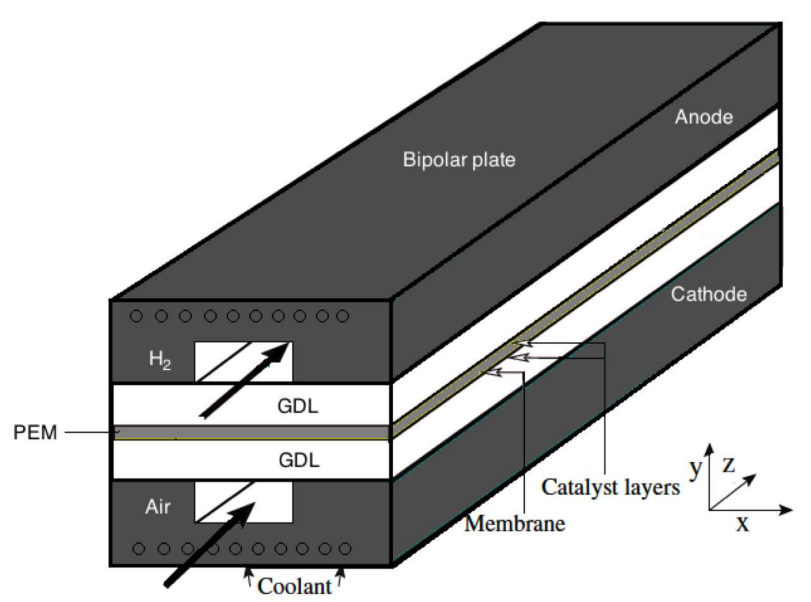

Figure 1: Single-channel PEMFC representation

\section{Mathematical modelling}

The simulation model is a $1+1 \mathrm{D}$ or quasi two-dimensional distributed parameters model [8]. It describes the different layers of the typical PEMFC: gas channels, gas diffusion layers (GDL), catalyst layers (CL) and the electrolyte membrane. Fig. 2 shows the problem modelling domain studied for the present work. Moreover, the model [8] includes the total current of the system and the potential drop in the membrane. The dynamics of the gas flows are described by partial derivatives in one direction, coupled to the reactions and transports through the membrane electrode assembly (MEA), which are represented as lumped parameters perpendicular to the gas flows, hence the $1+1 \mathrm{D}$ denomination of the model. The study assumptions include an isothermal model for the PEMFC. All modelling variables are collected and defined in Table 1.

Both anode and cathode gas channels follow mass balance equations to model the gas transport in the $\mathrm{z}$-direction
Table 1: Nomenclature and units of physical properties and constants

\begin{tabular}{|c|c|c|}
\hline Parameter & Description & Unit \\
\hline$C$ & Volumetric capacitance & $\mathrm{C} \mathrm{V}^{-1} \mathrm{~m}^{-3}$ \\
\hline$c_{i}$ & Concentration of $i$-th gas & $\mathrm{mol} \mathrm{m}^{-3}$ \\
\hline$D$ & Diffusion coefficient & $\mathrm{m}^{2} \mathrm{~s}^{-1}$ \\
\hline$F$ & Faraday constant & $\mathrm{C} \mathrm{mol}^{-} 1$ \\
\hline$f^{V}$ & Surface enlargement factor & - \\
\hline$I$ & Electrical current & $\mathrm{A}$ \\
\hline$i$ & Current density & $\mathrm{A} \mathrm{m}^{-2}$ \\
\hline$i_{0}$ & Exchange current density & $\mathrm{A} \mathrm{m}^{-2}$ \\
\hline$K$ & Pressure drop coefficient & $\mathrm{m}^{2} \mathrm{~s}^{-1} \mathrm{~Pa}^{-1}$ \\
\hline$L$ & Fuel cell length & $\mathrm{m}$ \\
\hline$L_{x}$ & Gas channel width & $\mathrm{m}$ \\
\hline$L_{z}$ & Gas channel depth & $\mathrm{m}$ \\
\hline$\dot{n}_{i, i n}$ & Inlet molar flux of $i$-th gas & $\operatorname{mol~m}{ }^{-2} \mathrm{~s}^{-1}$ \\
\hline$\dot{n}_{i}$ & y-direction flux of $i$-th gas & $\mathrm{mol} \mathrm{m} \mathrm{m}^{-2} \mathrm{~s}^{-1}$ \\
\hline$n_{V o l}$ & Discretisation volumes & - \\
\hline$p$ & Pressure & $\mathrm{Pa}$ \\
\hline$R$ & Gas constant & $\mathrm{J} \mathrm{mol}^{-1} \mathrm{~K}^{-1}$ \\
\hline$r$ & Reaction rate & $\mathrm{mol} \mathrm{m} \mathrm{m}^{-2} \mathrm{~s}^{-1}$ \\
\hline$T$ & Temperature & $\mathrm{K}$ \\
\hline$v$ & Flow velocity & $\mathrm{m} \mathrm{s}^{-1}$ \\
\hline$\Delta \Phi$ & Electrical potential & $\mathrm{V}$ \\
\hline$\Delta z$ & Discretisation length & $\mathrm{m}$ \\
\hline$\delta$ & Thickness of the layer & $\mathrm{m}$ \\
\hline$\lambda_{\text {anode }}$ & Anode stoichiometry & - \\
\hline$\lambda_{\text {cathode }}$ & Cathode stoichiometry, & - \\
\hline
\end{tabular}

$$
\begin{aligned}
v(t) & =-K \frac{\partial p(t)}{\partial z}, \\
p(t) & =R T \sum_{i} c_{i}(t), \\
\frac{\partial c_{i}(t)}{\partial t} & =-\frac{\partial}{\partial z}\left(v(t) c_{i}(t)\right)-\frac{\dot{n}_{i}(t)}{\delta},
\end{aligned}
$$

where subscript $i$ stands for the reactant, being $i=\mathrm{H}_{2}$ the hydrogen index and $i=\mathrm{H}_{2} \mathrm{O}$ the water in the anode side index. At the cathode side of the PEMFC $i=\mathrm{N}_{2}$ denotes the nitrogen, $i=\mathrm{O}_{2}$ the oxygen and $i=\mathrm{H}_{2} \mathrm{O}$ the water index. Reaction and water molar flux densities $\dot{n}_{i}$ are perpendicular to the channels in the $\mathrm{y}$-direction and are modelled as lumped parameters.

The diffusion effect in the GDLs of the PEMFC is mod- 


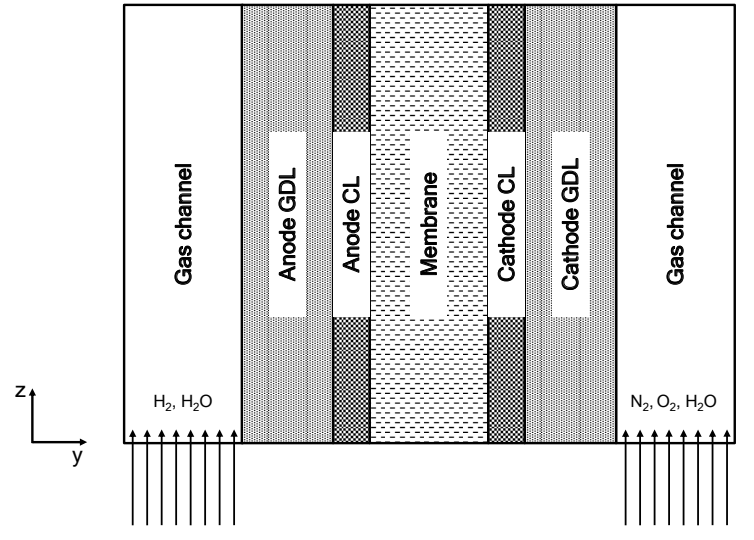

Figure 2: Problem modelling domain

elled adopting Fick's second law of diffusion

$$
\frac{\partial c_{i}(t)}{\partial t}=D_{i} \frac{\partial^{2} c_{i}(t)}{\partial y^{2}}
$$

with $D_{i}$ being the diffusion coefficient of the $i$-th gas species through the GDL (except for $\mathrm{N}_{2}$, which does not react).

The electrochemical reaction takes place in the CLs. The reacted molar fluxes are function of the anodic and cathodic reaction rates $r^{k}$, with $k=A$ for the anode side and $k=C$ for the cathode side. In the anode side, the amount of $\mathrm{H}_{2}$ consumed at the catalyst layer is

$$
\dot{n}_{H_{2}}(t)=r^{A}(t)
$$

Furthermore, considering that there is no mass storage capacity at the GLDs, it is possible to express the water transport from the anode gas channel to the membrane as the water transport towards the membrane

$$
\dot{n}_{H_{2} O}^{A}(t)=\dot{n}_{H_{2} O}^{A M}(t),
$$

where the superscript $A M$ specifies that it is a flux exiting from the anode side and entering into the electrolyte membrane.

At the cathode side, the electrochemical relation that governs the kinetics of the reaction is expressed as

$$
\dot{n}_{O_{2}}^{C}(t)=\frac{1}{2} r^{C}(t)
$$

The water transport from the cathode gas channel to the membrane through the GDL is

$$
\dot{n}_{\mathrm{H}_{2} \mathrm{O}}^{C}(t)=\dot{n}_{\mathrm{H}_{2} \mathrm{O}}^{C M}(t)-r^{C}(t) .
$$

Since the nitrogen is not a reactant in the PEMFC, if the cathode is fed with air, the nitrogen through the GDL vanishes. This is expressed as the following molar flux:

$$
\dot{n}_{N_{2}}^{C}=0
$$

Reaction rate expressions are derived from Butler-Volmer kinetics as introduced in [9]. The anodic reaction rate is formulated by

$$
r^{A}(t)=f^{V} \frac{i_{0}^{A}}{2 F}\left[\exp (\Omega(t)) \frac{p_{H_{2}}^{A}(t)}{p_{H_{2}, r e f}}-1\right],
$$

where

$$
\Omega(t)=\frac{2 F}{R T^{S}}\left(\Delta \Phi^{A}(t)-\Delta \Phi_{r e f}^{A}\right)
$$

The cathodic reaction rate can be written as

$$
r^{C}(t)=f^{V} \frac{i_{0}^{C}}{2 F} \exp (\Upsilon) \frac{p_{O_{2}}^{C}(t)}{p_{O_{2}, r e f}} \exp (\Sigma(t)),
$$

with

$$
\begin{aligned}
\Upsilon & =-\frac{\Delta G_{0}}{R}\left(\frac{1}{T^{S}}-\frac{1}{T_{r e f}}\right), \\
\Sigma(t) & =-\frac{\alpha 2 F}{R T^{S}}\left(\Delta \Phi^{C}(t)-\Delta \Phi_{r e f}^{C}\right) .
\end{aligned}
$$

A complete water transport model [10] is implemented in the membrane layer of the PEMFC. Water plays a key role in the dynamics of the proton transport. Water content in a PEMFC membrane can be defined as the relation between the number of water moles and the moles of polymer in the membrane. This parameter is also included in the membrane layer model.

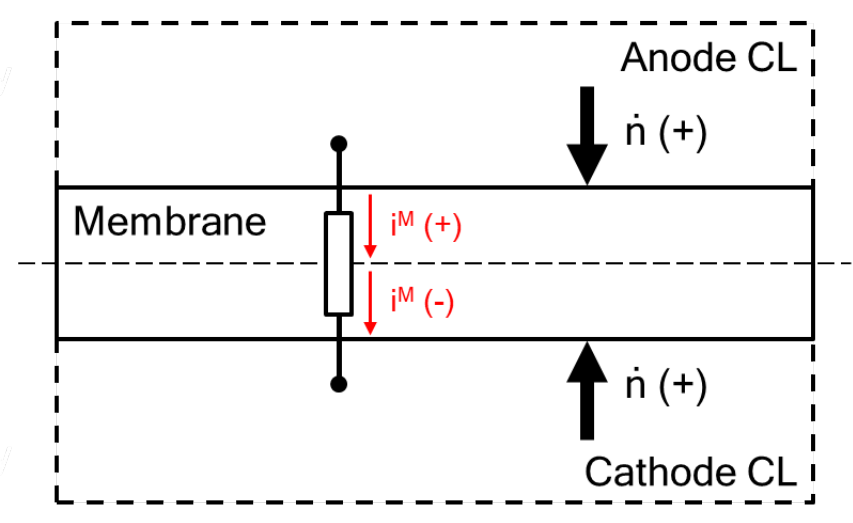

Figure 3: Sign criteria for the y-axis flows and currents

The electrical charge is transported through the membrane along the y-axis. The sign criteria is such that flows and currents are positive towards the membrane and negative in the opposite direction as shown in Fig. 3. The center of the membrane determines the switching between the reference frames. Hence, the signs for the charge balances are different at each side of the PEMFC, as expressed 
model

$$
\begin{aligned}
\Delta \dot{\Phi}_{j}^{A}(t) & =\frac{i_{j}^{M}(t)-2 F r_{j}^{A}(t)}{C^{A} \delta^{A C}}, \\
\Delta \dot{\Phi}_{j}^{C}(t) & =\frac{-i_{j}^{M}(t)+2 F r_{j}^{C}(t)}{C^{C} \delta^{C C}},
\end{aligned}
$$

where index $\mathrm{j}$ stands for the discretisation volume as it will be explained in Section 5 .

From Eq. (12), the potential differences $\Delta \Phi_{j}^{A}$ and $\Delta \Phi_{j}^{C}$ are obtained. Assuming that the fuel cell voltage $U$ is established beforehand, the total potential drop in the membrane can be expressed as

$$
\Delta \Phi_{j}^{M}(t)=\Delta \Phi_{j}^{C}(t)-\Delta \Phi_{j}^{A}(t)-U(t) .
$$

The total current for the PEMFC model is computed from the sum of all the currents through the membrane $\left(i_{j}^{M}\right)$, which is a function of the proton flow $\dot{n}_{H_{+}, j}$ :

$$
I(t)=L_{x} L_{z} \sum_{j}^{L_{z}} i_{j}^{M}(t)=L_{x} L z \sum_{j}^{L_{z}} F \dot{n}_{H_{+}, j}(t) .
$$

\section{Observation problem}

The observation of the gas species concentrations is going to be the focus of the study. These internal variables are a key aspect for the efficiency, durability, reliability and safety of the system. Knowing the concentration values along the z-axis is an essential feature in order to design and synthesise advanced controllers to improve the power quality and increase the performance of the PEMFC.

From the point of view of the mathematical complexity, the gas concentrations estimation is more accessible than other state variables in the model, like the charge balances. This is due to the additional complexity that the water model adds to the estimation process. Following the mass balance model presented in Eq. (1) and recalling that the reaction molar fluxes and water transport terms are measured disturbances in this paper, it can be concluded that the degree of dependency on the membrane water content of the gas concentrations model is lower than with the charge balances as depicted in Eqs. (8), (10) and (12). Thus, the estimation of the gas concentrations is less demanding.

Since only the estimation of the concentrations is considered, the observer internal model (which is different from the simulation model presented in Section 3) only includes these states. The observer model is described in Section 5.2. Moreover, the simplified observer internal model improves the convergence time and reduces the model complexity in the proposed solution.

Taking into account the generic nonlinear state space

$$
\begin{aligned}
& \dot{\mathbf{x}}(t)=\mathbf{f}(t, \mathbf{x}(t), \mathbf{u}(t)), \\
& \mathbf{y}(t)=\mathbf{h}(t, \mathbf{x}(t), \mathbf{u}(t)),
\end{aligned}
$$

where $\mathbf{x} \in \mathbb{R}^{n}$ is the state vector, $\mathbf{y} \in \mathbb{R}^{p}$ the output vector and $\mathbf{u} \in \mathbb{R}^{m}$ the input vector, for the observer model the states are the gas species concentrations $\dot{c}_{i}$, the measured outputs are the concentrations at the end of the cathode and anode gas channels and the inputs are the inlet flows, reaction and water transport terms, which are considered to be measured too. Therefore $x_{i}(t) \triangleq c_{i}(t)$, where $i$ refers to the $i$-th gas concentrations as discussed in Section 3, the output vector $y$ can be defined as the five gas species concentrations output measurements

$$
\mathbf{y}(t)=\left[\begin{array}{c}
h_{H_{2}}^{A}(\mathbf{x}) \\
h_{H_{2} O}^{A}(\mathbf{x}) \\
h_{N_{2}}^{C}(\mathbf{x}) \\
h_{O_{2}}^{C}(\mathbf{x}) \\
h_{H_{2} O}^{C}(\mathbf{x})
\end{array}\right]=\left[\begin{array}{c}
c_{H_{2}, \text { out }(t)}^{A} \\
c_{H_{2} O, \text { out }(t)}^{A} \\
c_{N_{2}, \text { out }(t)}^{C} \\
c_{\mathrm{O}_{2}, \text { out }(t)}^{C} \\
c_{\mathrm{H}_{2} O, \text { out }(t)}^{C}
\end{array}\right],
$$

and the input vector $u$ includes all the molar fluxes (inlet flows, reaction and water transport terms), hence $u(t) \triangleq$ $\left[\dot{n}_{i, i n}, \dot{n}_{i}(t)\right]$.

\section{Nonlinear distributed parameter observer syn- thesis}

As introduced in Section 4, the observation of the gas species concentrations $c_{i}$ along the gas channels is the main focus of this paper.

\subsection{Finite-difference discretisation}

It is possible to implement a finite-difference discretisation in order to solve numerically the spatial derivatives of the model presented in Section 3 with the aid of a computer and a mathematical solver. Finite-difference discretisation plays a special role when known boundary conditions are present in the problem. For the case study, the model presents several boundary conditions, such as the input molar fluxes or the external ambient pressure at the end of the gas channels. Henceforth, forward or backward differences will be applied to take advantage from the aforesaid boundary conditions.

The forward difference of a spatial derivative for a generic $x$-dependant function $f(x)$ is expressed as

$$
\frac{\partial f(x)}{\partial x}=\frac{\Delta_{x}[f](x)}{\Delta x}=\frac{f(x+\Delta x)-f(x)}{\Delta x},
$$

while the backward difference follows the expression

$$
\frac{\partial f(x)}{\partial x}=\frac{\nabla_{x}[f](x)}{\Delta x}=\frac{f(x)-f(x-\Delta x)}{\Delta x} .
$$

The model equations presented in Section 3 are discretised applying Eqs. (17) and (18) to implement the NDPO 
and to apply lumped parameter theory to synthesise the observer.

\subsection{Observer model}

Rewriting Eq. (1) in state space representation (see Eq. (15a)), and taking into account that the states of the system are the concentration values $\left(x_{i}(t) \triangleq c_{i}(t)\right)$, it is possible to write

$$
\begin{aligned}
v^{k}(t) & =-K^{k} \frac{\partial p^{k}(t)}{\partial z} \\
p^{k}(t) & =R T^{k} \sum_{i} x_{i}^{k}(t), \\
\dot{x}_{i, j}^{k}(t) & =-\frac{\partial}{\partial z}\left(v^{k}(t) x_{i}^{k}(t)\right)-\frac{\dot{n}_{i}^{k}(t)}{\delta^{k}},
\end{aligned}
$$

where superscript $k$ is included, with $k=A$ for the anode and $k=C$ for the cathode side of the PEMFC.

To take advantage of the boundary conditions of the problem, a backward difference discretisation, as shown in Eq. (18), is applied to the gas flow velocity Eq. (19a), while the forward difference discretisation presented in Eq. (17) is applied to the pressure term in Eq. (19b).

Subsequently, applying the aforementioned discretisation procedure to Eq. (19), the following discretised dynamic model for both gas channels is obtained:

$$
\begin{aligned}
v_{j}^{k}(t) & =\frac{K^{k}}{\Delta z}\left(p_{j}^{k}(t)-p_{j+1}^{k}(t)\right) \\
p_{j}^{k}(t) & =R T^{k} \sum_{i} x_{i, j}^{k}(t) \\
\dot{x}_{i, j}^{k}(t) & =\frac{v_{j-1}^{k}(t) x_{i, j-1}^{k}(t)}{\Delta z}-\frac{v_{j}^{k}(t) x_{i, j}^{k}(t)}{\Delta z}-\frac{\dot{n}_{i, j}^{k}(t)}{\delta^{k}},
\end{aligned}
$$

where subscript $j$ specifies the spatially-discretised finite volume.

The boundary conditions are given by $v_{j-1}(t) x_{i, j-1}(t)=$ $\dot{n}_{i, i n}(t) \triangleq u_{i}(t)$ for the first volume of discretisation $(j=1)$ and $p_{j+1}=p^{a m b}$ for the last discretisation boundary $(j=$ $\left.n_{V o l}\right)$. Applying these boundary conditions to Eq. (20) results in the state space equations for the first, middle and last discretisation volumes that can be expressed as

$\dot{x}_{i, j}^{k}(t)= \begin{cases}\frac{\dot{n}_{i, i n}^{k}(t)}{\Delta z}-\zeta_{1}^{k} \Psi^{k}(j)-\frac{\dot{n}_{i, j}^{k}(t)}{\delta^{k}}, & \text { if } j=1, \\ \zeta_{1}^{k}\left(\Psi^{k}(j-1)-\Psi^{k}(j)\right)-\frac{\dot{n}_{i, j}^{k}(t)}{\delta^{k}}, & \text { if } 2 \leq j \leq n_{V} \\ \zeta_{1}^{k} \Psi^{k}(j-1)+\Gamma^{k}(j+1)-\frac{\dot{n}_{i, j}^{k}(t)}{\delta^{k}}, & \text { if } j=n_{V o l},\end{cases}$

where

$$
\begin{aligned}
\Psi^{k}(j) & =x_{i, j}^{k}(t)\left(\sum_{i} x_{i, j}^{k}(t)-\sum_{i} x_{i, j+1}^{k}(t)\right), \\
\Psi^{k}(j-1) & =x_{i, j-1}^{k}(t)\left(\sum_{i} x_{i, j-1}^{k}(t)-\sum_{i} x_{i, j}^{k}(t)\right),
\end{aligned}
$$

being $\zeta_{1}^{k}=K R T / \Delta z^{2}$ and $\zeta_{2}^{k}=K p^{a m b} / \Delta z^{2}$. These spatially-discretised equations will be used for the development of the NDPO.

When developing the observer, the implementation complexity will limit the total discretisation volumes $\left(n_{V o l}\right)$ depending on the conditions of the overall problem. In Fig. 4 the main structure of the discretised PEMFC is presented for the $n$-discretised finite elements generalisation.

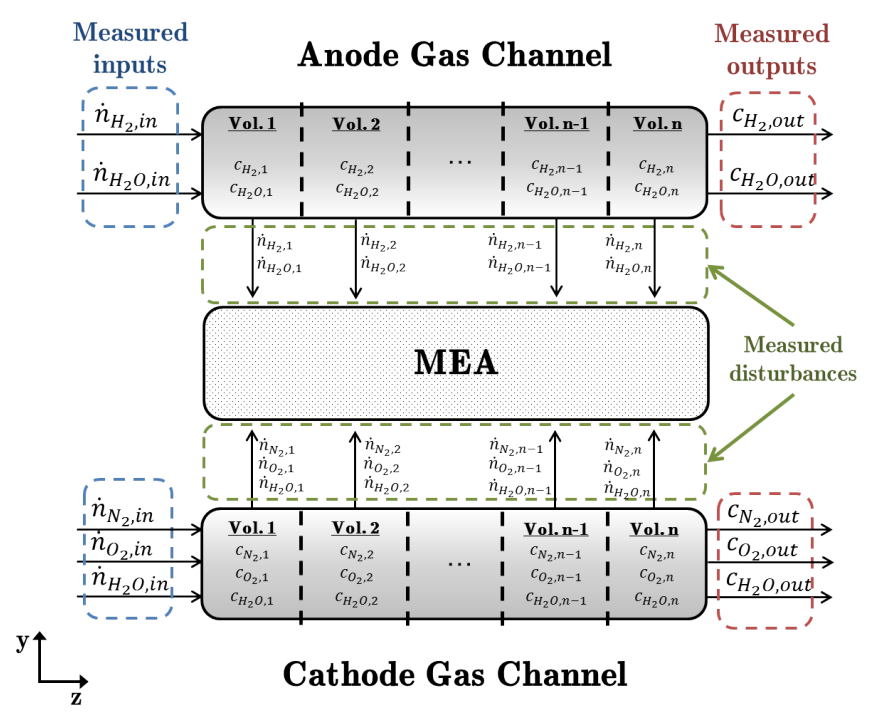

Figure 4: Generalised model of the discretised PEMFC

\subsection{Measurements}

As depicted in Fig. 4, the final concentration values at the end of the anode and cathode gas channels are considered the measured outputs. In a real implementation, the direct measurement of these variables would imply the use of sensors that are expensive and slow. However, the output concentration values can be inferred from other accessible measurements.

Employing a humidity sensor, the water partial pressure at the anode output $\left(p_{\mathrm{H}_{2} \mathrm{O} \text {, out }}^{A}\right)$ is obtained. From this measurement, it is possible to infer the water concentration as shows Eq. (20b). Moreover, knowing the total output pressure $\left(p_{\text {out }}^{A}\right)$ at the anode output gas channel, which is common in the majority of the PEMFC-based systems, the $\mathrm{H}_{2}$ partial pressure is obtained as

$$
p_{H_{2}, \text { out }}^{A}(t)=p_{\text {out }}^{A}(t)-p_{H_{2} O, \text { out }}^{A}(t),
$$

from which the $\mathrm{H}_{2}$ concentration at the end of the anode gas channel is derived.

Since at the cathode side there is an additional gas in comparison with the anode side, an extra equation is needed to obtain the output concentrations. With a humidity sensor the output water concentration can be obtained.

From the measurement of the total current demanded by the load $I(t)$, the total reacted $\mathrm{O}_{2}$ flow rate is obtained 
$[11]$

$$
\dot{n}_{O_{2}, r}^{C}(t)=L_{x} L_{z} \frac{I(t)}{4 F} .
$$

In this paper, the input molar flows are considered as measured variables (see Fig. 4). From the reacted $\mathrm{O}_{2}$ in Eq. (24) and using the $\mathrm{O}_{2}$ input molar flow $\left(\dot{n}_{\mathrm{O}_{2}, \text { in }}^{C}\right)$ measurement, the total output $\mathrm{O}_{2}$ molar flow is computed

$$
\dot{n}_{O_{2}, \text { out }}^{C}(t)=\dot{n}_{O_{2}, \text { in }}^{C}(t)-\dot{n}_{O_{2}, r}^{C}(t),
$$

which represents the flux of oxygen per second at the output of the cathode gas channel. To obtain the value of the output $\mathrm{O}_{2}$ concentration, Eq. (25) is divided by the gas flow velocity $v^{C}$, which can be obtained through the flowrate and total pressure at the channel input:

$$
c_{O_{2}, \text { out }}^{C}(t)=\frac{\dot{n}_{O_{2}, \text { out }}^{C}(t)}{v^{C}(t)} .
$$

Obtaining $c_{O_{2} \text { out }}^{C}$ through these steps would include a certain error because the internal dynamics of the channel are neglected. However, this fact would only affect the observation procedure during a short transitory.

Once the output concentrations of $\mathrm{H}_{2} \mathrm{O}$ and $\mathrm{O}_{2}$ are computed, the partial pressures can be obtained from Eq. (20b) Moreover, knowing $p_{\mathrm{H}_{2}, \text { out }}^{C}, p_{\mathrm{O}_{2}, \text { out }}^{C}$ and $p_{\text {out }}^{C}$, the partial pressure of $\mathrm{N}_{2}$ is obtained. Finally, the nitrogen concentration is derived using the previously computed values.

\subsection{Structure of the observer}

The main structure of the NDPO follows a model-based approach extracted from the literature [7]. For an $n$-order nonlinear system the observer structure is such that

$$
\begin{aligned}
\dot{\hat{\mathbf{x}}}(t) & =\mathbf{f}(t, \hat{\mathbf{x}}(t), \mathbf{u}(t))+\mathbf{g}(\hat{\mathbf{x}}(t)) \mathbf{u}_{\mathbf{0}}(t), \\
\hat{\mathbf{y}}(t) & =\mathbf{h}(t, \hat{\mathbf{x}}(t)),
\end{aligned}
$$

where the generalised observed state vector $\dot{\hat{\mathbf{x}}} \in \mathbb{R}^{n}$ and the observed output variable $\hat{\mathbf{y}} \in \mathbb{R}^{p}$. The function $\mathbf{f}(\hat{\mathbf{x}}, \mathbf{u})$ contains the nonlinear dynamics of the model presented in Eq. (21). The decoupling vector $\mathbf{g}(\hat{\mathbf{x}})$ provides the observer with full relative degree $n$ with respect to the input vector $\mathbf{u}$ from Eq. (15). The correction input $\mathbf{u}_{\mathbf{0}}$ is designed to achieve null estimation error in a finite amount of time. In the present work, a super-twisting algorithm (STA) based in SMC control techniques [3] is implemented. Since the STA does not depend on the model as much as other solutions (i.e. Model Predictive Control), the complexity of the observer, which already uses a nonlinear model, is not increased due to the implementation of the corrective action. Moreover, the STA approach provides robustness to the overall solution. Henceforth, it is appropriate to implement this technique as the corrective action term for the NDPO.
The NDPO observer inputs are the molar flows of the PEMFC-based system and the output measurements of the PEMFC shown in Eq. (16). The observer gives as an output the full state profile of the concentration values at each discretisation volume (see Fig. 5).

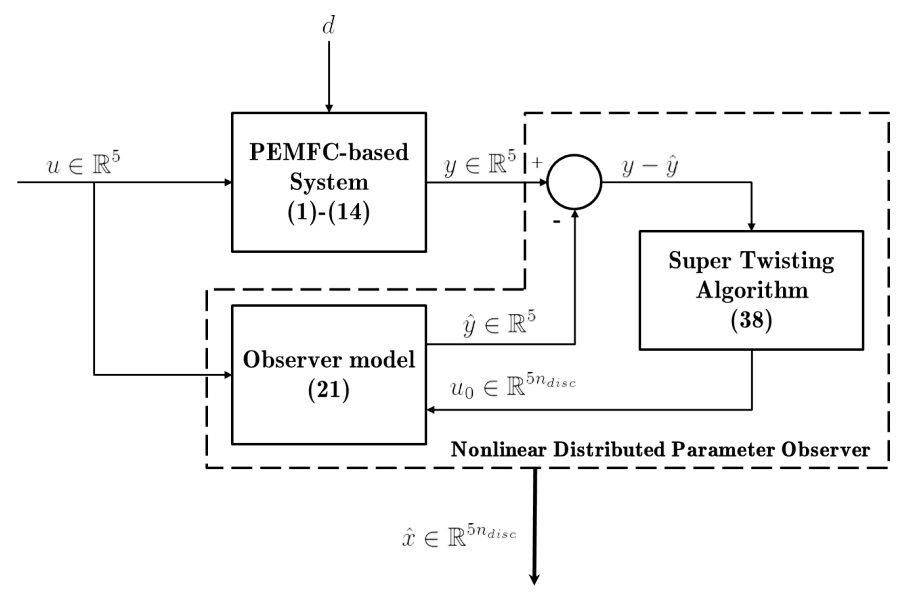

Figure 5: NDPO topology for the case study

\subsection{Nonlinear observability and design conditions}

For the nonlinear case, the observability condition arises from the computation of the observability matrix $\mathcal{O}$ that maps the outputs of the system to the initial state values by computing all the repeated Lie derivatives [12] of the output vector field $\mathbf{y}$. It can be expressed as

$$
\mathcal{O}(\mathbf{x})=\frac{\partial}{\partial \mathbf{x}}\left[\begin{array}{c}
h(\mathbf{x}) \\
L_{\mathbf{f}(\mathbf{x})} h(\mathbf{x}) \\
\vdots \\
L_{\mathbf{f}(\mathbf{x})}^{(n-2)} h(\mathbf{x}) \\
L_{\mathbf{f}(\mathbf{x})}^{(n-1)} h(\mathbf{x})
\end{array}\right],
$$

where the Lie derivative can be written as the tensor field defined by

$$
L_{\mathbf{f}(\mathbf{x})} \mathbf{h}(\mathbf{x})=\frac{\partial \mathbf{h}(\mathbf{x})}{\partial \mathbf{x}} \mathbf{f}(\mathbf{x}) .
$$

The full state vector is observable if Eq. (28) is nonsingular, meaning that it has full rank. The observability matrix (28) strongly depends on the state vector $\mathbf{x}$. As a result of this dependency, the analytical computation of the rank of Eq. (28) is a demanding task. In this paper the observability condition study has been performed numerically: The observability matrix was developed for the case study of $n_{V o l}=5$ and the computation of the rank was done through the singular value decomposition of the observability matrix. If during the simulation $\operatorname{rank}(\mathcal{O}(\mathbf{x}))$ is lower than the expected full rank, the observer is disconnected until the observability is recovered.

The output observation error is defined as the difference between the estimated output $\hat{y}$ of the observer (27) 
and the measured vector $y$ as expressed by

$$
e_{y}(t)=\hat{y}(t)-y(t)
$$

While the state observation error is written as

$$
e(t)=\hat{x}(t)-x(t)=\left[\begin{array}{c}
e_{1}(t) \\
e_{2}(t) \\
\vdots \\
e_{n}(t)
\end{array}\right]
$$

And given the output error vector

$$
\varepsilon(t)=\left[\begin{array}{c}
e_{y}(t) \\
\dot{e}_{y}(t) \\
\vdots \\
e_{y}^{n-1}(t)
\end{array}\right]
$$

it is demonstrated in [7] that the observer (27) is able to estimate the full state vector of system (21) (and drive the state observation error (31) to zero) in finite time given that a suitable correction action $u_{0}$ is selected to drive the output error vector (32) to zero in finite time. In this paper a STA approach is proposed for this task. The general equation for the STA $[3,13]$ can be expressed as

$$
\begin{aligned}
u(t) & =u_{1}(t)+\dot{u}_{2}(t), \\
u_{1}(t) & =\left\{\begin{array}{cc}
-K_{1}\left|s_{0}\right|^{\rho} \operatorname{sign}(s), & \text { if }|s|>\left|s_{0}\right|, \\
-K_{1}|s|^{\rho} \operatorname{sign}(s), & \text { if }|s| \leq\left|s_{0}\right|,
\end{array}\right. \\
\dot{u}_{2}(t) & =-K_{2} \frac{1}{2} \operatorname{sign}(s),
\end{aligned}
$$

where $K_{1}, K_{2}$ and $\rho$ are tuning constants of the correction algorithm. The sufficient conditions for the finite time convergence of Eq. (33) to the observation error sliding surface are defined as $[13,14]$

$$
\begin{aligned}
K_{1}^{2} & \geq \frac{4 \Lambda}{\Gamma_{m}^{2}} \frac{\Gamma_{M}\left(K_{2}+\Lambda\right)}{\Gamma_{m}\left(K_{2}-\Lambda\right)}, \\
K_{2} & >\frac{\Lambda}{\Gamma_{m}}, \\
0 & <\rho \leq 0.5,
\end{aligned}
$$

being $\Gamma_{M}, \Gamma_{m}$ and $\Lambda$ the upper and lower bounds for the sliding surface and the constant for the sliding region respectively as developed in [14].

\subsection{Implementation of the observer for the PEMFC model}

The design of the gas species concentrations observer is developed from the main observer structure shown in Eq. (27), having confirmed numerically the observability condition presented in Eq. (28) beforehand.

As depicted in Eq. (21), if the input molar flows $\dot{n}_{i, i n}^{k}$ are considered as measured inputs of the system and the value of the reaction and water transport terms are measured disturbances, mathematically it is possible to iso- late the overall observation problem (27) into two subproblems, each one defined by the corresponding gas channel model (21) and decoupled from each other (see Fig. 4)

$$
\dot{\hat{\mathbf{x}}}(t)=\left\{\begin{array}{l}
\mathbf{f}\left(t, \hat{\mathbf{x}}^{A}(t), \mathbf{u}^{A}(t)\right)+\mathbf{g}\left(\hat{\mathbf{x}}^{A}(t)\right) \mathbf{u}_{\mathbf{0}}{ }^{A}(t) \\
\mathbf{f}\left(t, \hat{\mathbf{x}}^{C}(t), \mathbf{u}^{C}(t)\right)+\mathbf{g}\left(\hat{\mathbf{x}}^{C}(t)\right) \mathbf{u}_{\mathbf{0}}{ }^{C}(t)
\end{array}\right\},
$$

with $\mathbf{x}=\left[\mathbf{x}^{A} ; \mathbf{x}^{C}\right]^{T}=\left[x_{i, j}^{A} ; x_{i, j}^{C}\right]^{T}$. The decoupling vector $\mathbf{g}=\left[\mathbf{g}^{A} ; \mathbf{g}^{C}\right]^{T}$ is defined in Eq. (37) for each side of the PEMFC.

This simplification of the observation problem allows to improve the performance of the observer and to obtain faster convergence times to the states.

The output vectors for the anode and cathode gas channels were defined in Eq. (16). From this, the anode and cathode observability matrices $\left(\mathcal{O}^{A}\left(\mathbf{x}^{k}\right)\right.$ and $\left.\mathcal{O}^{C}\left(\mathbf{x}^{k}\right)\right)$ can be developed following Eq. (28). Considering the output vectors at each side of the PEMFC, the conditions to guarantee the observability of each observation problem are:

$$
\begin{aligned}
& \operatorname{rank}\left(\mathcal{O}^{A}\left(\mathbf{x}^{k}\right)\right)=2 n_{V o l}, \\
& \operatorname{rank}\left(\mathcal{O}^{C}\left(\mathbf{x}^{k}\right)\right)=3 n_{V o l} .
\end{aligned}
$$

Function $\mathbf{f}\left(t, \hat{\mathbf{x}}^{\mathbf{k}}(t), \mathbf{u}^{k}(t)\right)$ includes the nonlinear dynamic description presented in Eq. (1). The vector $\mathbf{g}^{k}\left(\hat{\mathbf{x}}^{k}\right)$ is obtained from the observability matrices $\mathcal{O}^{k}\left(\mathbf{x}^{k}\right)$ introduced in Eq. (36) by computing [7]

$$
\mathbf{g}^{k}\left(\hat{\mathbf{x}}^{k}(t)\right)=\left(\mathcal{O}^{k}\left(\mathbf{x}^{k}\right)\right)^{-1}\left(\hat{\mathbf{x}}^{k}(t)\right)[0,0, \ldots, 1]^{T} .
$$

To remove the observation error, a corrective input $\mathbf{u}_{\mathbf{0}}$ is included in the main observer structure. As mentioned before, it is based in a STA control law from [3], and is expressed as

$$
\begin{aligned}
u_{0, i}^{k}(t) & =-K_{1}\left|e_{y}\right|^{\rho} \operatorname{sign}\left(e_{y}(t)\right)+v(t), \\
\dot{v}(t) & =-K_{2} \frac{1}{2} \operatorname{sign}\left(e_{y}(t)\right),
\end{aligned}
$$

with the output observation error (30) as the sliding variable and $K_{1}, K_{2}$ and $\rho$ tuning constants of the corrective action law as defined in Eq. (34). The fine tuning of these parameters is out of the scope of the present paper.

\section{Simulation results}

The initial state for all simulations is defined by the vector $\mathbf{x}_{\mathbf{0}} \in \mathbb{R}^{5 \times n_{V o l}}$ (with $n_{V o l}=5$ for the case study of this paper), which denotes the five gas species present in both gas channels. The observer is initialised with an initial observed state vector $\hat{\mathbf{x}}_{\mathbf{0}}=\mathbf{0} \in \mathbb{R}^{5 \times n_{V o l}}$. Simulations have been carried out using Simulink for MATLAB ${ }^{\circledR}$ R2011a (32 bits), running in a PC Intel ${ }^{\circledR}$ Core $^{\text {TM }}$ i7-3770 at $3.40 \mathrm{GHz}$ with $8 \mathrm{~GB}$ of RAM.

\subsection{Simulation scenario}

The simulation starts at a steady operating point under the conditions presented in Table 2. From these operation 
condition, the input molar fluxes for the gas species are computed. The resulting values are shown in Table 3.

Table 2: Initial operating conditions for the simulation

\begin{tabular}{lcc}
\hline Condition & Unit & Value \\
\hline Anode stoichiometry & - & 2.2 \\
Cathode stoichiometry & - & 3 \\
Fuel cell temperature & $\mathrm{K}$ & 353 \\
Cell voltage & $\mathrm{V}$ & 0.5 \\
\hline
\end{tabular}

Table 3: Input molar fluxes

\begin{tabular}{lc}
\hline Input molar flow & Value $\left[\mathrm{mol} \mathrm{m}^{-2} \mathrm{~s}^{-1}\right]$ \\
\hline $\mathrm{H}_{2}$ & 16 \\
$\mathrm{H}_{2} \mathrm{O}$ (anode) & 4 \\
$\mathrm{~N}_{2}$ & 224 \\
$\mathrm{O}_{2}$ & 60 \\
$\mathrm{H}_{2} \mathrm{O}$ (cathode) & 3 \\
\hline
\end{tabular}

Step changes are applied to the molar fluxes at simulation times $t=125 \mathrm{~s}, t=250 \mathrm{~s}$ and $t=375 \mathrm{~s}$ as presented in Table 4 . The aim of these step changes is to test the dynamical behaviour of the observer when the concentration values fluctuate in the gas channels as the system gets driven far away from its initial operating point.

Table 4: Step values for the dynamical analysis

\begin{tabular}{lcc}
\hline Input molar flow & Time $[\mathrm{s}]$ & Initial $\Rightarrow$ Final $\left[\mathrm{mol} \mathrm{m}^{-2} \mathrm{~s}^{-1}\right]$ \\
\hline $\mathrm{O}_{2}$ & 125 & $60 \Rightarrow 30$ \\
$\mathrm{H}_{2}$ & 250 & $16 \Rightarrow 14$ \\
$\mathrm{H}_{2} \mathrm{O}$ (anode) & 375 & $4 \Rightarrow 3$ \\
\hline
\end{tabular}

\subsection{Results and discussion}

Even though the estimation of the gas concentration species is done for all discretisation volumes in order to obtain a detailed profile, the figures only show the estimation at the middle point of the gas channel. It is virtually impossible to install sensors in this section of the PEMFC, therefore, the results obtained in this section have substantial relevance.

As it can be extracted from Figs. 6 and 7, the observation of the gas species concentration (dashed lines in the figures) is performed properly in a simulation environment. For the reconstruction of the anode gas channel gases, the convergence occurs in less than 0.5 seconds, while in the cathode side the observed states converge even faster. The difference between the state reconstruction times depend

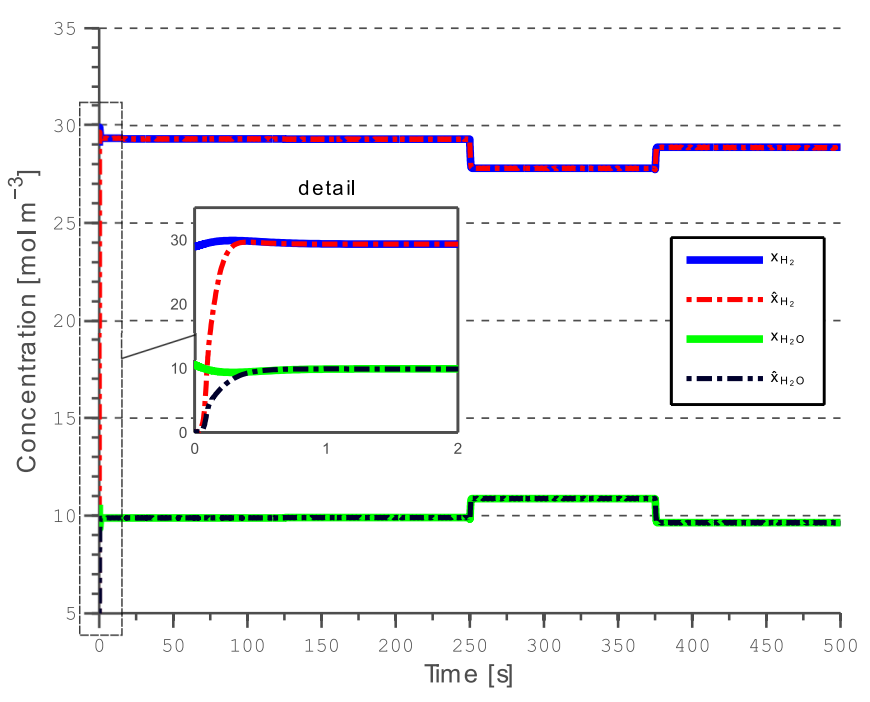

Figure 6: State estimation in the anode gas channel

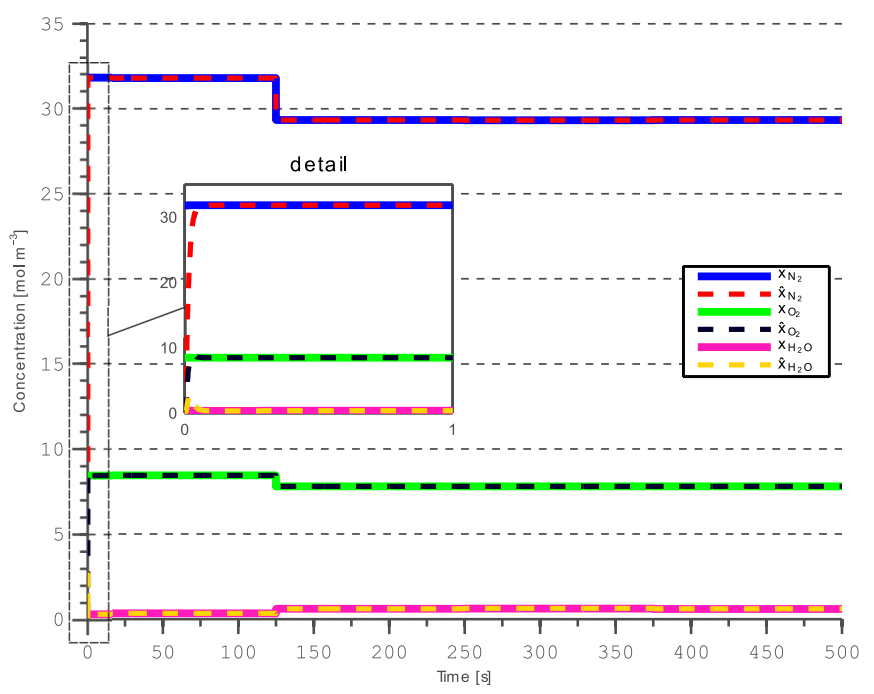

Figure 7: State estimation in the anode gas channel

on several conditions such as the $K_{1}$ and $K_{2}$ gains of the STA corrective action, the initial observed state vector $\hat{\mathbf{x}}_{\mathbf{0}}$ or the initial values of the real states of the system. Moreover, the reaction dynamics are different for each side of the PEMFC as evidenced in Eqs. (8) and (10). After the observed states converge to the real values, the estimation error is negligible even after the step changes for the dynamic analysis are injected to the PEMFC.

\subsection{Robustness analysis}

The results presented in Section 6.2 do not consider uncertainty. To test the robustness of the observer proposed in this paper, three new simulations in the presence of unknown inputs and model uncertainties are carried out.

To begin with, a simulation in the presence of input uncertainty is carried out. In particular, a uniform random signal between \pm 0.6 is multiplied to the water transport terms at the anode side of the PEMFC $\left(\dot{n}_{H_{2} O, j}^{A}\right)$. At 
the presence of this unknown input the observer behaves similarly as in Figs. 6 and 7 but with a slower initial convergence to the states.

The second simulation to test the robustness of the NDPO considers that the PEMFC-based system is under the effect of a model uncertainty $d$ (see Fig. 5). Specifically, the temperature of the system is considered to be under the effect of band-limited white noise with a $\pm 15 \%$ band limit from the original temperature value. As it is shown in Fig. 8, the observer is able to track perfectly the states under the presence of this uncertainty in the plant.

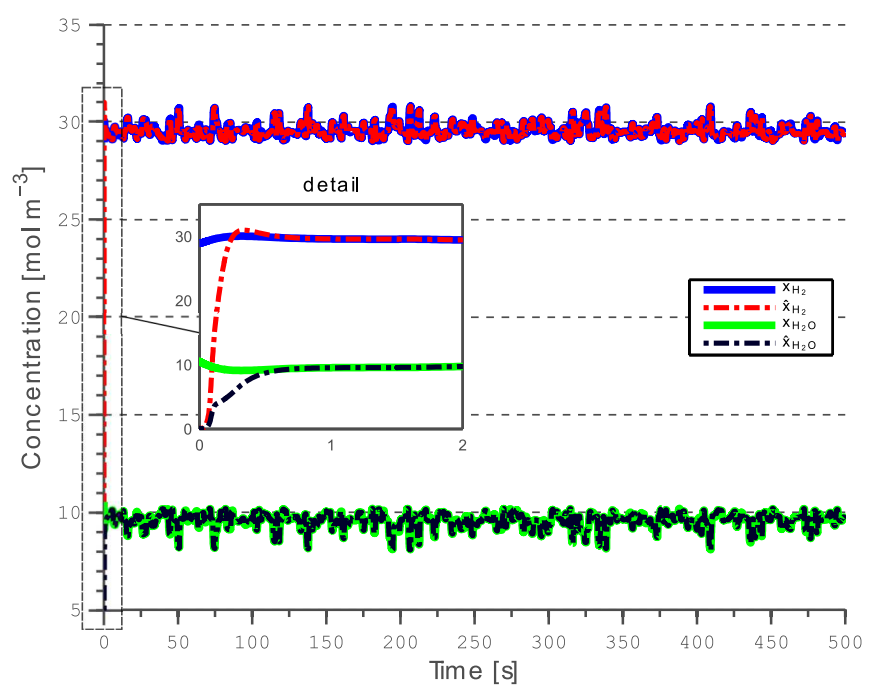

Figure 8: State estimation in the anode gas channel in the presence of model uncertainties

Finally, a simulation model that considers the delays associated with the measurement of the input molar flows and output concentrations has been used to test the observer behaviour in front of these changes. A pure delay of 15 seconds has been considered for the measured outputs of the system (a common value for commercial humidity sensors). For the input measurements, a delay of 1 second has been considered since mass flow meters operate with delays around 1 second. In Fig. 9, the effect of these measurement delays on the estimation is shown when the input molar fluxes change their value abruptly according to Table 4 . It can be seen that the convergence to the real value, which depends on the input measurements, is practically achieved within few seconds, obtaining appropriate state estimation even in the presence of the output measurement delay of 15 seconds. An oscillation before stabilisation appears (shown in the detail), which is produced by the output measurements delay, however, it has a small overshoot. Therefore, the observer performs correctly with the measurement delays and it does not introduce important estimation delays that could hinder closed-loop stability.

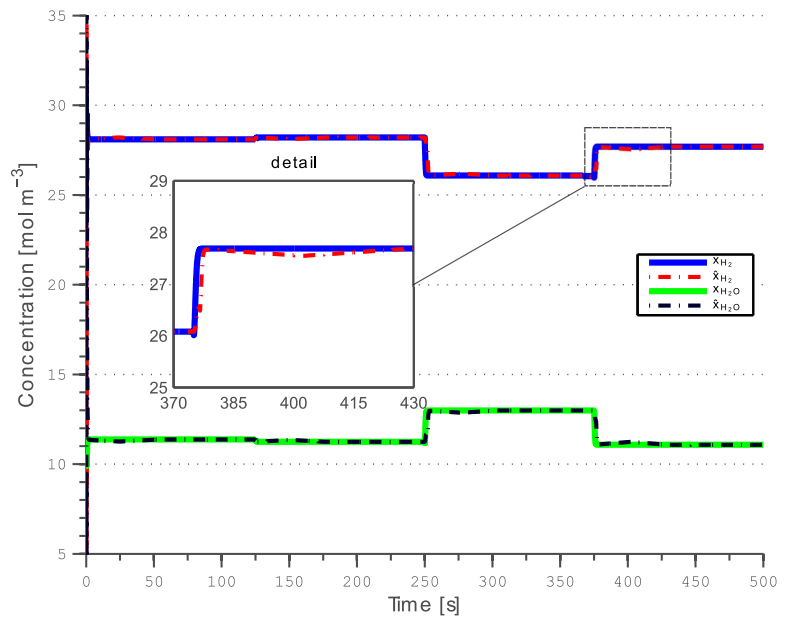

Figure 9: State estimation in the anode gas channel in the presence of time delays

\section{Conclusions}

This paper describes the design of a nonlinear observer for a PEMFC energy system. The proposed observer, synthesised for a system that is described by a distributed parameter model, allows the recovering of unknown information about the internal and unmeasurable state variables. The performance of the observer has been evaluated, obtaining satisfactory observation results of the anode and cathode gas concentrations for specific simulation scenarios even in the presence of uncertainty.

During the development of the observer, the observability matrix of the system was introduced. Through the inversion of the aforesaid matrix, the observer decoupling term is computed to reconstruct the full state vector from the output concentration measurements.

In this paper, the reaction and water transport terms have been considered as measured disturbances. The continuation of the research includes the consideration of the model and input uncertainties in the design of the observer. Furthermore, the design and implementation of a disturbance observer is currently under consideration to remove the aforementioned assumption.

A total of five discretisation volumes have been taken into consideration to perform the simulations. It is possible to generalise the results to a higher number of volumes to obtain more detailed concentration profiles. Nevertheless, the increase of discretisation volumes introduces complexity when synthesising the observer. A trade-off situation arises between the required level of detail of the recovered state information and the mathematical complexity of obtaining the observer.

\section{Acknowledgements}

This work is partially funded by the Spanish national MICINN project DPI2011-25649, as well as by the 7th 
Framework Programme of the European Commission in the context of the Fuel Cells and Hydrogen Joint Undertaking (FCH JU) through the project PUMA-MIND FP7 303419 .

\section{References}

[1] C. H. Lien, Robust observer-based control of systems with state perturbations via LMI approach, IEEE Transactions on Automatic Control 49 (8) (2004) pp. 1365-1370.

[2] H. H. Choi, M. J. Chung, Robust observer-based $\mathcal{H}_{\infty}$ controller design for linear uncertain time-delay systems, Automatica 33 (9) (1997) pp. 1749-1752.

[3] W. Perruquetti, J. P. Barbot, Sliding mode control in engineering, CRC Press, 2002.

[4] H. Görgün, M. Arcak, F. Barbir, An algorithm for estimation of membrane water content in PEM fuel cells, Journal of Power Sources 157 (1) (2006) pp. 389-394.

[5] I. Kazmi, A. Bhatti, S. Iqbal, A nonlinear observer for PEM fuel cell system, in: IEEE 13th International Multitopic Conference, Islamabad, Pakistan, 2009, pp. 1-6.

[6] M. Arcak, H. Görgün, L. M. Pedersen, S. Varigonda, A nonlinear observer design for fuel cell hydrogen estimation, IEEE Transactions on Control Systems Technology 12 (1) (2004) pp. 101-110.

[7] J. Davila, L. Fridman, A. Pisano, E. Usai, Finite-time state observation for non-linear uncertain systems via higher-order sliding modes, International Journal of Control 82 (8) (2009) pp. 1564-1574.

[8] M. Mangold, A. Bück, R. Hanke-Rauschenbach, Passivity based control of a distributed PEM fuel cell model, Journal of Process Control 20 (3) (2010) pp. 292-313.

[9] M. Wöhr, K. Bolwin, W. Schnurnberger, M. Fischer, W. Neubrand, G. Eigenberger, Dynamic modelling and simulation of a polymer membrane fuel cell including mass transport limitation, International Journal of Hydrogen Energy 23 (3) (1998) pp. 213-218.

[10] W. Neubrand, Modellbildung und Simulation von Elektromembranverfahren, Logos-Verlag, 1999.

[11] F. Barbir, PEM fuel cells: theory and practice, Elsevier Academic Press, 2013.

[12] J. J. E. Slotine, W. Li, et al., Applied nonlinear control, Vol. 199, Prentice-Hall Englewood Cliffs, NJ, 1991.

[13] A. Levant, Sliding order and sliding accuracy in sliding mode control, International Journal of Control 58 (6) (1993) pp. 1247 1263.

[14] C. Kunusch, P. F. Puleston, M. A. Mayosky, J. Riera, Sliding mode strategy for PEM fuel cells stacks breathing control using a super-twisting algorithm, IEEE Transactions on Control Systems Technology 17 (1) (2009) pp. 167-174. 